\title{
ON THE LATTICE OF FACES OF THE $n$-CUBE ${ }^{1}$
}

\author{
BY N. METROPOLIS AND GIAN-CARLO ROTA
}

Communicated by J. R. Goldman, August 8, 1977

1. We give a simple axiom system for the lattice $L_{n}$ of faces of the $n$-cube, which is independent of dimension, and we construct a partition of the lattice into a minimum number of chains, or Dilworth partition. This partition turns out to enjoy some notable symmetries.

We use the representation of the faces of an $n$-cube as signed subsets of an $n$-set, say of the set $\{1,2, \ldots, n\}$. A signed subset $A_{\sigma}=\left(A_{1}, A_{2}\right)$ is an ordered pair of disjoint subsets, where $A_{1}$ is called the positive part, and $A_{2}$ the negative-part. If $B_{\sigma}$ is also a signed set, write $A_{\sigma} \leqslant B_{\sigma}$ when $A_{1} \supseteq B_{1}$ and $A_{2}$ $\supseteq B_{2}$. Add a minimum element 0 -the improper face-to the ordered set of signed sets, thereby making it a lattice $L_{n}$. The maximum element $I$ of $L_{n}$ is the signed set $I=(\phi, \phi)$. We use the terms "face" and "signed set" interchangeably.

On the lattice of signed subsets one defines diagonals $\Delta\left(A_{\sigma}, \cdot\right)$. For a given face $A_{\sigma}$, such a diagonal is a function defined on the segment $\left[0, A_{\sigma}\right]$ of $L_{n}$, and $\Delta\left(A_{\sigma}, B_{\sigma}\right)=C_{\sigma}$, where $C_{1}=\left(A_{1}, B_{2}\right)$ and $C_{2}=\left(A_{2}, B_{1}\right)$. On the improper face one sets $\Delta\left(A_{\sigma}, 0\right)=0$. Geometrically, the diagonal $\Delta\left(A_{\sigma}, \cdot\right)$ associates to each face contained in $A_{\sigma}$ the unique opposite face inside the face $A_{\sigma}$. When $A_{\sigma}=I$, the diagonal $\Delta(I, \cdot)$, written $\Delta\left({ }^{\cdot}\right)$, is a cubical analog of complementation in a Boolean algebra.

2. Main Theorem. Let $L$ be a finite lattice with minimum 0 and maximum I. For every $x \neq 0$, let $\Delta_{x}$ be a function defined on the segment $[0, x]$ and taking values in $[0, x]$. Assume: (1) if $y \leqslant x$, then $\Delta_{x}\left(\Delta_{x}(y)\right)=y$; (2) if $a \leqslant b$ $\leqslant x$, then $\Delta_{x}(a) \leqslant \Delta_{x}(b)$; (3) if $a<x$, then $a \wedge \Delta_{x}(a)=0$; (4) let $a<x$ and $b<x$. Then the following two conditions are equivalent: $\Delta_{x}(a) \wedge b<x$ and $a \wedge b=0$. Then $L$ is isomorphic to the lattice of faces of an $n$-cube for some $n$, and conversely.

3. A symmetric Dilworth partition. By Dilworth's theorem there exists a partition of $L_{n}$ into [n/3] chains. We explicitly describe one such partition, one that is invariant under the main diagonal $\Delta$.

Consider a signed subset as a sequence $u_{1} u_{2} \cdots u_{n}$ whose digits $u_{i}$ range over the alphabet $\{x, 0,1\}$. Set $u_{i}=x$ if the element $i$ is unsigned; otherwise,

AMS (MOS) subject classifications (1970). Primary 05 B40; Secondary 05 A17.

1 Work supported by US Energy Research and Development Administration under Contract W-7405-ENG. 36 and partially supported by NSF MCS 7701947.

Copyright (c) 1978, American Mathematical Society 
$u_{i}=0$ or 1 according to whether $u_{i}$ belongs to the positive or to the negative parts. We call 0 and 1 significant digits in contrast to $x$. A vertex is a sequence $u_{1} u_{2} \cdots u_{n}$ where no $u_{i}=x$.

The chains issuing from the vertices are constructed one step at a time, upwards from the vertices, according to the following rule:

(1) Change the last two digits of the vertex $u_{1} u_{2} \cdots u_{n}$, according to the following table: $11 \rightarrow 1 x, 00 \rightarrow 0 x, 10 \rightarrow x 0,01 \rightarrow x 1$. Denote the resulting face by $v_{1} v_{2} \cdots v_{n}$.

(2) Change the two rightmost adjacent significant digits of $v_{1} v_{2} \cdots v_{n}$ by the same rule.

(3) Repeat until the resulting face contains no two adjacent significant digits.

(4) Now the preceding algorithm assigns to chains issuing from vertices all faces $u_{1} u_{2} \cdots u_{n}$ for $u_{1}=u_{2}=x$, where $u_{j}$ is a significant digit for some $j>2$, and no two successive digits are significant.

(5) For faces containing three successive digits $x 00, x 01, x 10$ or $x 11$ use the following bracketing algorithm: (a) Bracket $x 01$ to ( $x 01)$, etc., by inserting brackets at the appropriate places. In the resulting bracketed face, two digits are successive if they are successive in the sequence obtained after all bracketed digits have been removed. (b) Repeat the bracketing algorithm (a), for successive digits, until no unbracketed digits can be bracketed, and obtain a completely bracketed face. (c) On the set of completely bracketed faces having the same bracketed digits and the same bracket structure, remove bracketed digits and proceed as in steps (1)-(4), and then reinsert the bracketed digits in their original places. One obtains in this way a partition into chains of such a set. (d) The only remaining faces to assign to chains are those which have only $x$ 's in their unbracketed sequence, which is nonempty.

Let $u=u_{1} u_{2} \cdots u_{n}$ be one of these remaining faces. If $u=I$, the maximum element, assign it arbitrarily to any chain, say, the chain containing $0 x x$ $\cdots x$. If $u \neq I$, let $u_{k}$ be the first significant digit, and let $u_{m}$ be the first unbracketed digit, which must be an $x$. Then assign $u$ to the chain containing $v_{1} v_{2}$ $\cdots v_{n}$ where $v_{m}=u_{k}$ and $v_{i}=u_{i}$ otherwise.

The preceding technique for constructing Dilworth partitions can be extended to families of $k$ disjoint subsets, ordered similarly to the case $k=2$ of the cube, and even to certain sublattices of the lattice of partitions of a set. These constructions will be presented elsewhere.

\section{BIBLIOGRAPHY}

Harper and Rota, Matching theory, an introduction, Advances in Probability, Vol. 1 (Peter Ney, editor), Dekker, New York, 1970, pp. 171-217. 
C. Greene and D. Kleitman, Proof techniques in the theory of finite sets, MAA Survey in Combinatorics (to appear).

UNIVERSITY OF CALIFORNIA, LOS ALAMOS SCIENTIFIC LABORATORY, LOS ALAMOS, NEW MEXICO 87545

DEPARTMENT OF MATHEMATICS, MASSACHUSETTS INSTITUTE OF TECHNOLOGY, CAMBRIDGE, MASSACHUSETTS 02139 